\title{
La potenza del gioco
}

\section{Stefania Manetti}

Pediatra di famiglia, Piano di Sorrento (Napoli)
Nel giocare il bambino diventa una spanna più alto. Come nell'osservare il punto centrale di una lente di ingrandimento il GIOCO contiene tutte le componenti dello sviluppo in una forma condensata ed è esso stesso una risorsa principale per lo sviluppo.

LEOV VYGOTSKY

Il gioco è un'attività semplice e allo stesso tempo estremamente profonda per un bambino. Il gioco rende naturale e gioioso l'apprendimento.

Da più di trent'anni la fondazione del Minnesota Children's Museum produce ricerca sul gioco e lo sviluppo dei bambini attraverso i contesti di apprendimento attivo e interattivo dei programmi del $\mathrm{mu}-$ seo. "Stimolare l'apprendimento attraverso il gioco" è il principio della Fondazione, esso si basa sulle seguenti affermazioni: "L'apprendimento precoce è la base dell'apprendimento di tutto il corso della vita. Le famiglie sono i primi maestri dei nostri bambini. Tutti i bambini hanno bisogno di tempo e di spazio per essere bambini. Le diverse prospettive arricchiscono la vita dei bambini. Il gioco è apprendere".

La potenza del gioco sta nel giocare sempre, ovunque e intensamente, anche senza giocattoli, senza genitori che at tivamente lo incoraggiano, anche nel bel mezzo di una zona di guerra. Talmente potente è il gioco da divenire una componente importante dello sviluppo del bambino; l'AAP (American Academy of Pediatrics) nel 2007 ha prodotto un documento sulla necessità del gioco, la Commissione ONU sui diritti umani ha riconosciuto il gioco un diritto fondamentale di ogni bambino.

Paradossalmente, mentre la ricerca scientifica accumula evidenze sulla importanza del gioco, i bambini di oggi giocano circa 8 ore in meno a settimana rispetto ai bambini di vent'anni fa. I motivi sono diversi, un ruolo consistente riguarda la pressione costante sugli standard accademici che ha visto il gioco sostituito da test per le scuole primarie e a volte anche nelle scuole della infanzia. A loro volta i genitori investono maggiormente in giochi definiti educativi. Tutto questo ha creato una dicotomia tra gioco e apprendimento.

Il documento prodotto dal gruppo di esperti del Minnesota Children's Museum fornisce una panoramica della ricerca scientifica mediante il principio filosofico che il gioco è apprendimento.

Con il gioco il bambino acquisisce l'abilità di regolare il comportamento, di costruire le fondamenta per apprendere la scienza e la matematica, per risolvere e negoziare le relazioni sociali complesse, costruire una serie di abilità atte a risolvere i problemi. Il documento descrive il ruolo degli adulti nel guidare i bambini attraverso le opportunità di giocare e apprendere. Gli adulti sono intuitivamente consapevoli di cosa si intenda per gioco, mentre per gli studiosi non esiste ancora una definizione formale. Forse la migliore è quella di Stuart Brown, il fondatore del National Institute for Play, che descrive il gioco come: "Qualsiasi attività fatta spontaneamente per se stessa, ossia un'attività che "apparentemente non ha scopi, produce piacere e gioia e porta il bambino alla fase successiva di padronanza”.

Il gioco è piacevole e non ha funzioni estrinseche o scopi, i bambini giocano per la soddisfazione di giocare. Durante il gioco per un bambino è più importante come si gioca rispetto al fine del gioco, esso è scelto liberamente ed è finzione. Secondo alcuni autori l'aspetto da considerare maggiormente è proprio la mancanza di scopo, che dà al gioco la qualità di "giocoso".

In opposizione al gioco, il lavoro non è visto come piacevole ed è costituito da motivazioni estrinseche, ossia ha uno scopo. Alcuni ricercatori hanno suggerito che forme ibride di lavoro e gioco possono fornire contesti ottimali e vantaggiosi per apprendere. Un esempio è il bambino impegnato in un lavoro scolastico difficile, con degli obbiettivi didattici precisi, che trova una sua motivazione intrinseca e un coinvolgimento. A metà di questo percorso, dove il gioco e il lavoro si intersecano, la motivazione del bambino, se affiancata dalla guida attenta dell'adulto possono creare un contesto di apprendimento giocoso.

\section{II ruolo del gioco libero e del gioco guidato dall'adulto}

Nel gioco guidato l'adulto facilita l'apprendimento del bambino che gioca attraverso delle interazioni.

Il ruolo dell'adulto nel gioco guidato può assumere due forme:
- l'adulto può arricchire il contesto di gioco del bambino fornendo esperienze o giochi che lo promuovono;

- l'adulto può "costruire" insieme al bambino un'impalcatura (scaffolding) partecipando al gioco come cogiocatore mediante domande e commentando le scoperte o incoraggiando nuove esplorazioni o aspetti del gioco. L'apprendimento attraverso il gioco in questi casi è più strutturato, ma deve sempre porre al centro il bambino e scaturire dal suo desiderio.

Per i lettori di Quaderni acp potrebbe emergere un'assonanza con il programma Nati per Leggere, in particolare con la lettura dialogica, dove l'adulto, in base al desiderio del bambino, si pone come facilitatore, amplificando il processo di lettura attraverso domande aperte e osservazioni reciproche al divertimento durante la lettura di una storia, ponendo sempre al centro il bambino e cercando di stimolare la sua curiosità. Non c'è apprendimento senza emozione, emozione come indiretta forma di conoscenza, in quanto non richiede informazioni.

Sia il gioco libero che quello guidato sono elementi essenziali. Il gioco libero dà al bambino autonomia vera, il gioco guidato è un percorso attraverso il quale i genitori e gli educatori possono fornire esperienze di apprendimento mirate. Durante il gioco i bambini sviluppano abilità di pensiero critico, emozioni, abilità sociali e fisiche. Il gioco promuove lo sviluppo cerebrale, e le competenze e le abilità acquisite nei primi anni di vita attraverso il gioco costruiscono le fondamenta per l'apprendimento futuro, dall'educazione primaria fino al posto di lavoro.

Nel gioco tutti i domini dello sviluppo - cognitivo, sociale, emotivo e fisico - sono coinvolti con benefici indiretti e diretti. Sono apprese e messe in pratiche competenze cognitive come il linguaggio, la capacità di risolvere problemi, la creatività e l'autoregolazione. Inoltre quando giocando i bambini apprendono come negoziare, raggiungere un compromesso e interagire con gli altri, si sviluppano le relazioni tra pari e la crescita emotiva; il bambino impara a gestire la rabbia, la paura e la fru- 
strazione. L'impegno fisico nel gioco, il manipolare le costruzioni, il disegno, la corsa, contribuiscono poi allo sviluppo di abilità motorie fini e grossolane.

Se il bambino ha l'opportunità di veicolare l'apprendimento attraverso il gioco questa occasione risponde in maniera immediata ai suoi bisogni di sviluppo e può contribuire alla scoperta di attività consone al proprio stile di apprendimento.

$\mathrm{Nel}$ gioco libero il bambino acquisisce il controllo del proprio processo di apprendimento, promuovendo il desiderio, la motivazione e la padronanza. Il bambino apprende anche a cercare la conoscenza, il gioco coinvolge l'esplorazione, il testare delle ipotesi. Tutto ciò facilitato da un contesto libero da pericoli, sicuro, privo di ansie, dove i bambini possono mettere a confronto e testare le proprie abilità. Ma il gioco è anche resilienza di fronte alle sfide che il giocare pone, contribuendo a sviluppare le fondamenta per un apprendimento continuo.

Il secolo in cui viviamo è caratterizzato da una mole incredibile di informazioni e conoscenze, necessarie per poter far parte di una comunità; molte delle abilità sviluppate nella prima infanzia con il gioco possono essere cruciali per avere successo in futuro. In un'economia globale chi è parte di una forza lavoro deve essere socialmente adeguato e al contempo creativo. Le 6 C: Collaborazione, Comunicazione, $\mathrm{Co}-$ noscenza dei contenuti, pensiero Critico, Creatività e la "Confidence" ossia la sicurezza di sé nel possibile fallimento e riprova, sono essenziali per il futuro successo dei bambini. Non tutte queste abilità si acquisiscono a scuola, molte si apprendono con il gioco.

Il gioco sociale è quello intrapreso tra pari o con gli adulti. Le mamme sono le prime partner di gioco, fin dai primi mesi di vita con il cucù-tetè e via via con il gioco più complesso, i genitori aiutano il bambino nella costruzione delle fondamenta delle loro abilità, se si pongono come iniziatori del gioco, che in questi casi può essere anche più strutturato e sofisticato. Il genitore che osserva, dà l'avvio e sostiene, e aiuta il bambino nel pianificare il percorso per lo sviluppo del gioco tra pari.

Nel 1932 Mildred Parten, sociologa della Università del Minnesota, descrisse livelli di gioco sociale che oggi sono considerati ancora abbastanza validi per delineare la maturità nel gioco nei primi anni di vita di un bambino.

Il gioco solitario del bambino di 2-3 anni, il gioco parallelo dei bambini dopo i 3 anni coinvolti in attività di gioco simili ma separate, il gioco associativo quando i bambini giocano separatamente ma condividono, comunicano con gli altri riguardo la propria attività di gioco; il gioco cooperativo dei bambini più grandi che hanno un obbiettivo comune e giocano insieme per raggiungerlo. Secondo moderni studiosi queste categorie della Parten non sono strettamente legate all'età ma dipendono anche dai contesti. Ricerche recenti hanno infatti evidenziato come in un contesto familiare tra pari i bambini hanno la capacità di giocare in maniera cooperativa anche a 18 mesi, età in cui molti bambini sono capaci di condividere il gioco e i propri giocattoli o esprimere disappunto o rabbia se non approvano il comportamento degli altri nel gioco. Nel gioco tra pari i bambini stabiliscono tra di loro le regole del gioco ("tu sei la principessa, io sarò il mago... costruiremo una navicella spaziale per andare sulla Luna...") mettendo in pratica la negoziazione, la pianificazione e la cooperazione. Questo tipo di gioco è pieno di conflitti e i bambini apprendono come i loro desideri non sono uguali a quelli degli altri e come la frustrazione generata possa essere gestita.

\section{II gioco simbolico è l'attività cardine della prima infanzia}

Come pediatri consideriamo la comparsa del gioco simbolico uno dei parametri utili per definire l'appropriatezza dello sviluppo di un bambino.

Il gioco simbolico si manifesta intorno all'anno di vita ed è diretto verso sé stessi, poi verso gli altri. Intorno ai 18 mesi il bambino trasforma gli oggetti conferendo loro delle caratteristiche animate, successivamente una banana può diventare un telefono, una pentola un cappello... A 2 anni se un adulto giocando trasforma un pentolino in una vasca da bagno il bambino potrebbe riempirla di acqua.

Con lo sviluppo, il gioco simbolico passa poi dagli oggetti alla decontestualizzazione dagli stessi e comincia il gioco attraverso situazioni immaginarie. L'età prescolare è l'età d'oro del gioco simbolico che tuttavia secondo molti studiosi continua anche dopo, in età scolare, anche se spesso in "privato" o nel contesto dei media.

Le affermazioni forti e audaci di Vygotsky riguardo all'importanza del gioco simbolico nello sviluppo del bambino sono supportate da ricerche recenti che ne sottolineano l'importanza nello sviluppo delle abilità sociali, cognitive ed emozionali.

\section{doc.manetti@gmail.com}

Ginsburg KR, American Academy of Pediatrics Committee on Communications; American Academy of Pediatrics Committee on Psychosocial Aspects of Child and Family Health. The importance of play in promoting healthy child development and maintaining strong parent-child bonds. Pediatrics. 2007 Jan;119(1):182-91.

Parten MB. Social play among preschool children. J Abnorm Soc Psychol. 1933 Jul;28:13647.

Shonkoff JP, Phillips DA. From neurons to neighborhoods: The science of early childhood development. National Academy Press, 2011.

United Nations High Commission on $\mathrm{Hu}-$ man Rights. Convention on the rights of the child. 1989.

Https://www.childrensmuseums.org/.

Elkind D. The Power of Play: Learning What Comes Naturally. American Journal of Play. 2008 1(1):1-6. 